\title{
Blow up of positive initial-energy solutions for coupled nonlinear wave equations with degenerate damping and source terms
}

\section{Erhan Pişkin*}

\section{"Correspondence:}

episkin@dicle.edu.tr

Department of Mathematics, Dicle

University, Diyarbakır, 21280, Turkey

\begin{abstract}
In this work, we consider coupled nonlinear wave equations with degenerate damping and source terms. We will show the blow up of solutions in finite time with positive initial energy. This improves earlier results in the literature.

MSC: 35B44; 35L05
\end{abstract}

Keywords: blow up; coupled wave equations; degenerate damping

\section{Introduction}

In this work, we consider the following initial-boundary value problem:

$$
\begin{cases}u_{t t}+\left(|u|^{k}+|v|^{l}\right)\left|u_{t}\right|^{p-1} u_{t}=\operatorname{div}\left(\rho\left(|\nabla u|^{2}\right) \nabla u\right)+f_{1}(u, v), & (x, t) \in \Omega \times(0, T), \\ v_{t t}+\left(|v|^{\theta}+|u|^{\varrho}\right)\left|v_{t}\right|^{q-1} v_{t}=\operatorname{div}\left(\rho\left(|\nabla v|^{2}\right) \nabla v\right)+f_{2}(u, v), & (x, t) \in \Omega \times(0, T), \\ u(x, t)=v(x, t)=0, & (x, t) \in \partial \Omega \times(0, T), \\ u(x, 0)=u_{0}(x), \quad u_{t}(x, 0)=u_{1}(x), & x \in \Omega, \\ v(x, 0)=v_{0}(x), \quad v_{t}(x, 0)=v_{1}(x), & x \in \Omega,\end{cases}
$$

where $\Omega$ is a bounded domain with smooth boundary $\partial \Omega$ in $R^{n}(n=1,2,3) ; p, q \geq 1$, $k, l, \theta, \varrho \geq 0 ; f_{i}(\cdot, \cdot): R^{2} \longrightarrow R$ are given functions to be specified later.

In the case of $\rho=1$, equation (1.1) takes the form

$$
\left\{\begin{array}{l}
u_{t t}-\Delta u+\left(|u|^{k}+|v|^{l}\right)\left|u_{t}\right|^{p-1} u_{t}=f_{1}(u, v), \\
v_{t t}-\Delta v+\left(|v|^{\theta}+|u|^{\varrho}\right)\left|v_{t}\right|^{q-1} v_{t}=f_{2}(u, v) .
\end{array}\right.
$$

In [1] Rammaha and Sakuntasathien studied the global well posedness of the solution of problem (1.2). Agre and Rammaha [2] studied the global existence and the blow up of the solution of problem (1.2) for $k=l=\theta=\varrho=0$, and also Alves et al. [3] investigated the existence, uniform decay rates and blow up of the solution to systems. After that, the blow up result was improved by Houari [4]. Also, Houari [5] showed that the local solution obtained in [2] is global and decay of solutions.

When $k=l=\theta=\varrho=0$, equation (1.1) reduces to the following form:

$$
\left\{\begin{array}{l}
u_{t t}+\left|u_{t}\right|^{p-1} u_{t}=\operatorname{div}\left(\rho\left(|\nabla u|^{2}\right) \nabla u\right)+f_{1}(u, v), \\
v_{t t}+\left|v_{t}\right|^{q-1} v_{t}=\operatorname{div}\left(\rho\left(|\nabla v|^{2}\right) \nabla v\right)+f_{2}(u, v) .
\end{array}\right.
$$

\section{Springer}

(c) 2015 Pişkin; licensee Springer. This is an Open Access article distributed under the terms of the Creative Commons Attribution License (http://creativecommons.org/licenses/by/4.0), which permits unrestricted use, distribution, and reproduction in any medium, provided the original work is properly credited. 
Wu et al. [6] obtained the global existence and blow up of the solution of problem (1.3) under some suitable conditions. Also, Fei and Hongjun [7] considered problem (1.3) and improved the blow up result obtained in [6] for a large class of initial data in positive initial energy using some techniques as in Payne and Sattinger [8] and some estimates used firstly by Vitillaro [9]. Recently, Pişkin and Polat [10] studied the local and global existence, energy decay and blow up of the solution of problem (1.3).

In this work, we analyze the influence of degenerate damping terms and source terms on the solutions of problem (1.1). Blow up of the solution with positive initial energy was proved for $2(r+2)>\max \{k+p+1, l+p+1, \theta+q+1, \varrho+q+1\}$ by using the technique of [9] with a modification in the energy functional.

This work is organized as follows. In Section 2, we present some lemmas and the local existence theorem. In Section 3, the blow up of the solution is given.

\section{Preliminaries}

In this section, we shall give some assumptions and lemmas which will be used throughout this work. Let $\|\cdot\|$ and $\|\cdot\|_{p}$ denote the usual $L^{2}(\Omega)$ norm and $L^{p}(\Omega)$ norm, respectively.

Next, we give assumptions for problem (1.1).

(A1) $\rho$ is a positive $C^{1}$ function satisfying

$$
\rho(s)=b_{1}+b_{2} s^{m}, \quad m \geq 0,
$$

where $b_{1}, b_{2}$ are nonnegative constants and $b_{1}+b_{2}>0$.

(A2) For the nonlinearity, we suppose that

$$
\begin{cases}p, q \geq 1 & \text { if } n=1,2 \\ 1 \leq p, q \leq 5 & \text { if } n=3\end{cases}
$$

Concerning the functions $f_{1}(u, v)$ and $f_{2}(u, v)$, we take

$$
\begin{aligned}
& f_{1}(u, v)=a|u+v|^{2(r+1)}(u+v)+b|u|^{r} u|v|^{r+2}, \\
& f_{2}(u, v)=a|u+v|^{2(r+1)}(u+v)+b|v|^{r} v|u|^{r+2},
\end{aligned}
$$

where $a, b>0$ are constants and $r$ satisfies

$$
\begin{cases}-1<r & \text { if } n=1,2 \\ -1<r \leq 1 & \text { if } n=3\end{cases}
$$

According to the above equalities they can easily verify that

$$
u f_{1}(u, v)+v f_{2}(u, v)=2(r+2) F(u, v), \quad \forall(u, v) \in R^{2},
$$

where

$$
F(u, v)=\frac{1}{2(r+2)}\left[a|u+v|^{2(r+2)}+2 b|u v|^{r+2}\right] .
$$

We have the following result. 
Lemma 2.1 [11] There exist two positive constants $c_{0}$ and $c_{1}$ such that

$$
c_{0}\left(|u|^{2(r+2)}+|v|^{2(r+2)}\right) \leq 2(r+2) F(u, v) \leq c_{1}\left(|u|^{2(r+2)}+|v|^{2(r+2)}\right)
$$

is satisfied.

Lemma 2.2 (Sobolev-Poincaré inequality) [12] Let q be a number with $2 \leq q<\infty(n=1,2)$ or $2 \leq q \leq 2 n /(n-2)(n \geq 3)$, then there is a constant $C_{*}=C_{*}(\Omega, q)$ such that

$$
\|u\|_{q} \leq C_{*}\|\nabla u\| \quad \text { for } u \in H_{0}^{1}(\Omega) .
$$

Lemma 2.3 [13] Suppose that

$$
p \leq 2 \frac{n-1}{n-2}, \quad n \geq 3
$$

holds. Then there exists a positive constant $C>1$ depending on $\Omega$ only such that

$$
\|u\|_{p}^{s} \leq C\left(\|\nabla u\|^{2}+\|u\|_{p}^{p}\right)
$$

for any $u \in H_{0}^{1}(\Omega), 2 \leq s \leq p$.

Lemma 2.4 $E(t)$ is a nonincreasing function for $t \geq 0$ and

$$
\frac{d}{d t} E(t)=-\int_{\Omega}\left(|u|^{k}+|v|^{l}\right)\left|u_{t}\right|^{p+1} d x-\int_{\Omega}\left(|v|^{\theta}+|u|^{\varrho}\right)\left|v_{t}\right|^{q+1} d x .
$$

Proof Multiplying the first equation of (1.1) by $u_{t}$, the second equation by $v_{t}$, and integrating them over $\Omega$, then adding them together and integrating by parts, we obtain

$$
E(t)-E(0)=-\int_{0}^{t} \int_{\Omega}\left(\left(|u|^{k}+|v|^{l}\right)\left|u_{\tau}\right|^{p+1}+\left(|v|^{\theta}+|u|^{\varrho}\right)\left|v_{\tau}\right|^{q+1}\right) d x d \tau
$$

$$
\text { for } t \geq 0 \text {. }
$$

Next, we state the local existence theorem that can be established by combining arguments of $[1,10]$. Firstly, we give the definition of a weak solution to problem (1.1).

Definition 2.1 A pair of functions $(u, v)$ is said to be a weak solution of (1.1) on [0,T] if $u, v \in C\left([0, T] ; W_{0}^{1,2(m+1)}(\Omega) \cap L^{r+1}(\Omega)\right), u_{t} \in C\left([0, T] ; L^{2}(\Omega)\right) \cap L^{p+1}(\Omega \times(0, T))$ and $v_{t} \in$ $C\left([0, T] ; L^{2}(\Omega)\right) \cap L^{q+1}(\Omega \times(0, T))$. In addition, $(u, v)$ satisfies

$$
\begin{gathered}
\int_{\Omega} u^{\prime}(t) \phi d x-\int_{\Omega} u_{1}(t) \phi d x+\int_{\Omega}\left(\rho\left(|\nabla u|^{2}\right) \nabla u\right) \nabla \phi d x \\
\quad+\int_{0}^{t} \int_{\Omega}\left(|u|^{k}+|v|^{l}\right)\left|u^{\prime}\right|^{p-1} u^{\prime} \phi d x d \tau \\
=\int_{0}^{t} \int_{\Omega} f_{1}(u(\tau), v(\tau)) \phi d x d \tau,
\end{gathered}
$$




$$
\begin{gathered}
\int_{\Omega} v^{\prime}(t) \varphi d x-\int_{\Omega} v_{1}(t) \varphi d x+\int_{\Omega}\left(\rho\left(|\nabla v|^{2}\right) \nabla v\right) \nabla \varphi d x \\
\quad+\int_{0}^{t} \int_{\Omega}\left(|v|^{\theta}+|u|^{\varrho}\right)\left|v^{\prime}\right|^{q-1} v^{\prime} \varphi d x d \tau \\
=\int_{0}^{t} \int_{\Omega} f_{2}(u(\tau), v(\tau)) \varphi d x d \tau
\end{gathered}
$$

for all test functions $\phi \in W_{0}^{1,2(m+1)}(\Omega) \cap L^{p+1}(\Omega), \varphi \in W_{0}^{1,2(m+1)}(\Omega) \cap L^{q+1}(\Omega)$ and for almost all $t \in[0, T]$.

Theorem 2.1 (Local existence) Assume that (A1), (A2) and (2.1) hold. Then, for any initial data $u_{0}, v_{0} \in W_{0}^{1,2(m+1)}(\Omega) \cap L^{r+1}(\Omega)$ and $u_{1}, v_{1} \in L^{2}(\Omega)$, there exists a unique local weak solution $(u, v)$ of problem (1.1) (in the sense of Definition 2.1) defined in $[0, T]$ for some $T>0$, and satisfies the energy identity

$$
E(t)+\int_{0}^{t} \int_{\Omega}\left(\left(|u|^{k}+|v|^{l}\right)\left|u_{\tau}\right|^{p+1}+\left(|v|^{\theta}+|u|^{\varrho}\right)\left|v_{\tau}\right|^{q+1}\right) d x d \tau=E(0)
$$

where

$$
E(t)=\frac{1}{2}\left(\left\|u_{t}\right\|^{2}+\left\|v_{t}\right\|^{2}\right)+\frac{1}{2} \int_{\Omega}\left(P\left(|\nabla u|^{2}\right)+P\left(|\nabla v|^{2}\right)\right) d x-\int_{\Omega} F(u, v) d x,
$$

where $P(s)=\int_{0}^{s} \rho(\xi) d \xi, s \geq 0$.

\section{Blow up of solutions}

In this section, we are going to consider the blow up of the solution for problem (1.1).

Lemma 3.1 Suppose that (2.1) holds. Then there exists $\eta>0$ such that for any $(u, v) \in$ $\left(H^{2 m}(\Omega) \cap H_{0}^{m}(\Omega)\right) \times\left(H^{2 m}(\Omega) \cap H_{0}^{m}(\Omega)\right)$ the inequality

$$
\|u+v\|_{2(r+2)}^{2(r+2)}+2\|u v\|_{r+2}^{r+2} \leq \eta\left(\int_{\Omega}\left(P\left(|\nabla u|^{2}\right)+P\left(|\nabla v|^{2}\right)\right)\right)^{r+2}
$$

holds.

Proof The proof is almost the same as that of [11], so we omit it here.

In order to state and prove our result and for the sake of simplicity, we take $a=b=1$. We introduce the following:

$$
\begin{aligned}
& B=\eta^{\frac{1}{2(r+2)}}, \quad \alpha_{1}=B^{-\frac{r+2}{r+1},} \\
& E_{1}=\left(\frac{1}{2}-\frac{1}{2(r+2)}\right) \alpha_{1}^{2}, \quad E_{2}=\left(\frac{1}{2(m+1)}-\frac{1}{2(r+2)}\right) \alpha_{1}^{2},
\end{aligned}
$$

where $\eta$ is the optimal constant in (3.1).

The following lemma will play an essential role in the proof of our main result, and it is similar to the lemma used firstly by Vitillaro [9]. 
Lemma 3.2 [7] Assume that (A1) and (2.1) hold. Let $(u, v)$ be a solution of (1.1). Assume further that $E(0)<E_{1}$ and

$$
\left(\int_{\Omega}\left(P\left(\left|\nabla u_{0}\right|^{2}\right)+P\left(\left|\nabla v_{0}\right|^{2}\right)\right) d x\right)^{\frac{1}{2}}>\alpha_{1} .
$$

Then there exists a constant $\alpha_{2}>\alpha_{1}$ such that

$$
\begin{aligned}
& \left(\int_{\Omega}\left(P\left(|\nabla u|^{2}\right)+P\left(|\nabla v|^{2}\right)\right) d x\right)^{\frac{1}{2}}>\alpha_{2} \quad \text { for } t>0 \\
& \left(\|u+v\|_{2(r+2)}^{2(r+2)}+2\|u v\|_{r+2}^{r+2}\right)^{\frac{1}{2(r+2)}} \geq B \alpha_{2} \quad \text { for } t>0 .
\end{aligned}
$$

Theorem 3.1 Assume that (A1), (A2) and (2.1) hold. Assume further that

$$
2(r+2)>\max \{k+p+1, l+p+1, \theta+q+1, \varrho+q+1\} .
$$

Then any solution of problem (1.1) with initial data satisfying

$$
\left(\int_{\Omega}\left(P\left(\left|\nabla u_{0}\right|^{2}\right)+P\left(\left|\nabla v_{0}\right|^{2}\right)\right) d x\right)^{\frac{1}{2}}>\alpha_{1}, \quad E(0)<E_{2},
$$

cannot exist for all time.

Proof We suppose that the solution exists for all time and we reach a contradiction.

Set

$$
H(t)=E_{2}-E(t)
$$

By using (2.10) and (3.6) we get

$$
\begin{aligned}
0< & H(0) \leq H(t)=E_{2}-\frac{1}{2}\left(\left\|u_{t}\right\|^{2}+\left\|v_{t}\right\|^{2}\right) \\
& -\frac{1}{2} \int_{\Omega}\left(P\left(|\nabla u|^{2}\right)+P\left(|\nabla v|^{2}\right)\right) d x+\int_{\Omega} F(u, v) d x .
\end{aligned}
$$

From (3.4) and (2.4) we have

$$
\begin{aligned}
E_{2} & -\frac{1}{2}\left(\left\|u_{t}\right\|^{2}+\left\|v_{t}\right\|^{2}\right)-\frac{1}{2} \int_{\Omega}\left(P\left(|\nabla u|^{2}\right)+P\left(|\nabla v|^{2}\right)\right) d x+\int_{\Omega} F(u, v) d x \\
& \leq E_{2}-\frac{1}{2} \alpha_{1}^{2}+\frac{c_{1}}{2(r+2)}\left(\|u\|_{2(r+2)}^{2(r+2)}+\|v\|_{2(r+2)}^{2(r+2)}\right) \\
& \leq E_{1}-\frac{1}{2} \alpha_{1}^{2}+\frac{c_{1}}{2(r+2)}\left(\|u\|_{2(r+2)}^{2(r+2)}+\|v\|_{2(r+2)}^{2(r+2)}\right) \\
& \leq-\frac{1}{2(r+2)} \alpha_{1}^{2}+\frac{c_{1}}{2(r+2)}\left(\|u\|_{2(r+2)}^{2(r+2)}+\|v\|_{2(r+2)}^{2(r+2)}\right) \\
& \leq \frac{c_{1}}{2(r+2)}\left(\|u\|_{2(r+2)}^{2(r+2)}+\|v\|_{2(r+2)}^{2(r+2)}\right) .
\end{aligned}
$$


Combining (3.7) and (3.8) we have

$$
0<H(0) \leq H(t) \leq \frac{c_{1}}{2(r+2)}\left(\|u\|_{2(r+2)}^{2(r+2)}+\|v\|_{2(r+2)}^{2(r+2)}\right)
$$

We then define

$$
\Psi(t)=H^{1-\sigma}(t)+\varepsilon\left(\int_{\Omega} u u_{t} d x+\int_{\Omega} v v_{t} d x\right),
$$

where $\varepsilon$ is small to be chosen later and

$$
\begin{aligned}
0< & \sigma \leq \min \left\{\frac{r+1}{2(r+2)}, \frac{2 r+3-(k+p)}{2 p(r+2)}, \frac{2 r+3-(l+p)}{2 p(r+2)},\right. \\
& \left.\frac{2 r+3-(\varrho+q)}{2 q(r+2)}, \frac{2 r+3-(\theta+q)}{2 q(r+2)}\right\} .
\end{aligned}
$$

Our goal is to show that $\Psi(t)$ satisfies a differential inequality of the form

$$
\Psi^{\prime}(t) \geq \xi \Psi^{\zeta}(t), \quad \zeta>1
$$

This, of course, will lead to a blow up in finite time.

By taking a derivative of (3.10) and using equation (1.1) we obtain

$$
\begin{aligned}
\Psi^{\prime}(t)= & (1-\sigma) H^{-\sigma}(t) H^{\prime}(t)+\varepsilon\left(\left\|u_{t}\right\|^{2}+\left\|v_{t}\right\|^{2}\right) \\
& -\varepsilon \int_{\Omega}\left(\rho\left(|\nabla u|^{2}\right)|\nabla u|^{2}+\rho\left(|\nabla v|^{2}\right)|\nabla v|^{2}\right) d x \\
& -\varepsilon\left(\int_{\Omega} u\left(|u|^{k}+|v|^{l}\right) u_{t}\left|u_{t}\right|^{p-1} d x+\int_{\Omega} v\left(|v|^{\theta}+|u|^{\varrho}\right) v_{t}\left|v_{t}\right|^{q-1} d x\right) \\
& +\varepsilon \int_{\Omega}\left(u f_{1}(u, v)+v f_{2}(u, v)\right) d x \\
= & (1-\sigma) H^{-\sigma}(t) H^{\prime}(t)+\varepsilon\left(\left\|u_{t}\right\|^{2}+\left\|v_{t}\right\|^{2}\right)-\varepsilon b_{1}\left(\|\nabla u\|^{2}+\|\nabla v\|^{2}\right) \\
& -\varepsilon b_{2}\left(\|\nabla u\|_{2(r+2)}^{2(r+2)}+\|\nabla v\|_{2(r+2)}^{2(r+2)}\right)+\varepsilon\left(\|u+v\|_{2(r+2)}^{2(r+2)}+2\|u v\|_{r+2}^{r+2}\right) \\
& -\varepsilon\left(\int_{\Omega} u\left(|u|^{k}+|v|^{l}\right) u_{t}\left|u_{t}\right|^{p-1} d x+\int_{\Omega} v\left(|v|^{\theta}+|u|^{\varrho}\right) v_{t}\left|v_{t}\right|^{q-1} d x\right) .
\end{aligned}
$$

From the definition of $H(t)$, it follows that

$$
\begin{aligned}
-b_{2}( & \left.\|\nabla u\|_{2(r+2)}^{2(r+2)}+\|\nabla v\|_{2(r+2)}^{2(r+2)}\right) \\
= & 2(m+1) H(t)-2(m+1) E_{2}+(m+1)\left(\left\|u_{t}\right\|^{2}+\left\|v_{t}\right\|^{2}\right) \\
& +(m+1) b_{1}\left(\|\nabla u\|^{2}+\|\nabla v\|^{2}\right)-2(m+1) \int_{\Omega} F(u, v) d x
\end{aligned}
$$

Substituting (3.14) into (3.13), we obtain

$$
\begin{aligned}
\Psi^{\prime}(t)= & (1-\sigma) H^{-\sigma}(t) H^{\prime}(t)+\varepsilon(m+2)\left(\left\|u_{t}\right\|^{2}+\left\|v_{t}\right\|^{2}\right) \\
& +\varepsilon b_{1} m\left(\|\nabla u\|^{2}+\|\nabla v\|^{2}\right)
\end{aligned}
$$




$$
\begin{aligned}
& +2 \varepsilon(m+1) H(t)-2 \varepsilon(m+1) E_{2}+\varepsilon\left(1-\frac{m+1}{r+2}\right)\left(\|u+v\|_{2(r+2)}^{2(r+2)}+2\|u v\|_{r+2}^{r+2}\right) \\
& -\varepsilon\left(\int_{\Omega} u\left(|u|^{k}+|v|^{l}\right) u_{t}\left|u_{t}\right|^{p-1} d x+\int_{\Omega} v\left(|v|^{\theta}+|u|^{\varrho}\right) v_{t}\left|v_{t}\right|^{q-1} d x\right)
\end{aligned}
$$

In order to estimate the last two terms in (3.15), we make use of the following Young inequality:

$$
X Y \leq \frac{\delta^{k} X^{k}}{k}+\frac{\delta^{-l} Y^{l}}{l}
$$

where $X, Y \geq 0, \delta>0, k, l \in R^{+}$such that $\frac{1}{k}+\frac{1}{l}=1$. Consequently, applying the above inequality we have

$$
\int_{\Omega} u u_{t}\left|u_{t}\right|^{p-1} d x \leq \frac{\delta_{1}^{p+1}}{p+1}\|u\|_{p+1}^{p+1}+\frac{p \delta_{1}^{-\frac{p+1}{p}}}{p+1}\left\|u_{t}\right\|_{p+1}^{p+1}
$$

and therefore

$$
\begin{aligned}
\int_{\Omega}\left(|u|^{k}+|v|^{l}\right) u u_{t}\left|u_{t}\right|^{p-1} d x \leq & \frac{\delta_{1}^{p+1}}{p+1} \int_{\Omega}\left(|u|^{k}+|v|^{l}\right)|u|^{p+1} d x \\
& +\frac{p \delta_{1}^{-\frac{p+1}{p}}}{p+1} \int_{\Omega}\left(|u|^{k}+|v|^{l}\right)\left|u_{t}\right|^{p+1} d x .
\end{aligned}
$$

Similarly

$$
\int_{\Omega} \nu v_{t}\left|v_{t}\right|^{q-1} d x \leq \frac{\delta_{2}^{q+1}}{q+1}\|v\|_{q+1}^{q+1}+\frac{q \delta_{2}^{-\frac{q+1}{q}}}{q+1}\left\|v_{t}\right\|_{q+1}^{q+1},
$$

and therefore

$$
\begin{aligned}
\int_{\Omega} v\left(|v|^{\theta}+|u|^{\varrho}\right) v_{t}\left|v_{t}\right|^{q-1} d x \leq & \frac{\delta_{2}^{q+1}}{q+1} \int_{\Omega}\left(|v|^{\theta}+|u|^{\varrho}\right)|v|^{q+1} d x \\
& +\frac{q \delta_{2}^{-\frac{q+1}{q}}}{q+1} \int_{\Omega}\left(|v|^{\theta}+|u|^{\varrho}\right)\left|v_{t}\right|^{q+1} d x
\end{aligned}
$$

where $\delta_{1}, \delta_{2}$ are constants depending on the time $t$ that will be specified later. Therefore, (3.15) becomes

$$
\begin{aligned}
\Psi^{\prime}(t) \geq & (1-\sigma) H^{-\sigma}(t) H^{\prime}(t)+\varepsilon(m+2)\left(\left\|u_{t}\right\|^{2}+\left\|v_{t}\right\|^{2}\right)+\varepsilon b_{1} m\left(\|\nabla u\|^{2}+\|\nabla v\|^{2}\right) \\
& +2 \varepsilon(m+1) H(t)-2 \varepsilon(m+1) E_{2}+\varepsilon\left(1-\frac{m+1}{r+2}\right)\left(\|u+v\|_{2(r+2)}^{2(r+2)}+2\|u v\|_{r+2}^{r+2}\right) \\
& -\varepsilon \frac{\delta_{1}^{p+1}}{p+1} \int_{\Omega}\left(|u|^{k}+|v|^{l}\right)|u|^{p+1} d x-\varepsilon \frac{p \delta_{1}^{-\frac{p+1}{p}}}{p+1} \int_{\Omega}\left(|u|^{k}+|v|^{l}\right)\left|u_{t}\right|^{p+1} d x \\
& -\varepsilon \frac{\delta_{2}^{q+1}}{q+1} \int_{\Omega}\left(|v|^{\theta}+|u|^{\varrho}\right)|v|^{q+1} d x-\varepsilon \frac{q \delta_{2}^{-\frac{q+1}{q}}}{q+1} \int_{\Omega}\left(|v|^{\theta}+|u|^{\varrho}\right)\left|v_{t}\right|^{q+1} d x .
\end{aligned}
$$


Therefore, by taking $\delta_{1}$ and $\delta_{2}$ so that $\delta_{1}^{-\frac{p+1}{p}}=k_{1} H^{-\sigma}(t), \delta_{2}^{-\frac{q+1}{q}}=k_{2} H^{-\sigma}(t)$ where $k_{1}, k_{2}>0$ are specified later, we get

$$
\begin{aligned}
\Psi^{\prime}(t) \geq & ((1-\sigma)-K \varepsilon) H^{-\sigma}(t) H^{\prime}(t)+\varepsilon(m+2)\left(\left\|u_{t}\right\|^{2}+\left\|v_{t}\right\|^{2}\right)+\varepsilon b_{1} m\left(\|\nabla u\|^{2}+\|\nabla v\|^{2}\right) \\
& +2 \varepsilon(m+1) H(t)+\varepsilon c^{\prime}\left(\|u\|_{2(r+2)}^{2(r+2)}+\|v\|_{2(r+2)}^{2(r+2)}\right) \\
& -\varepsilon \frac{k_{1}^{-p} H^{\sigma p}(t)}{p+1} \int_{\Omega}\left(|u|^{k}+|v|^{l}\right)|u|^{p+1} d x \\
& -\varepsilon \frac{k_{2}^{-q} H^{\sigma q}(t)}{q+1} \int_{\Omega}\left(|v|^{\theta}+|u|^{\varrho}\right)|v|^{q+1} d x
\end{aligned}
$$

where $K=\frac{k_{1} p}{p+1}+\frac{k_{2} q}{q+1}$ and $c^{\prime}=c_{0}\left(1-\frac{m+1}{r+2}-2(m+1) E_{2}\left(B \alpha_{2}\right)^{-2(r+2)}\right)$. It is clear $c^{\prime}>0$ since $\alpha_{2}>\alpha_{1}=B^{-\frac{r+2}{r+1}}$.

Applying the Young inequality, we have

$$
\begin{aligned}
\int_{\Omega}\left(|u|^{k}+|v|^{l}\right)|u|^{p+1} d x \leq & \int_{\Omega}|u|^{k+p+1} d x+\int_{\Omega}|v|^{l}|u|^{p+1} d x \\
\leq & \int_{\Omega}|u|^{k+p+1} d x+\frac{l}{l+p+1} \delta_{1}^{\frac{l+p+1}{l}} \int_{\Omega}|v|^{l+p+1} d x \\
& +\frac{p+1}{l+p+1} \delta_{1}^{-\frac{l+p+1}{p+1}} \int_{\Omega}|u|^{l+p+1} d x \\
= & \|u\|_{k+p+1}^{k+p+1}+\frac{l}{l+p+1} \delta_{1}^{l+p+1}\|v\|_{l+p+1}^{l+p+1} \\
& +\frac{p+1}{l+p+1} \delta_{1}^{-\frac{l+p+1}{p+1}}\|u\|_{l+p+1}^{l+p+1}
\end{aligned}
$$

and

$$
\begin{aligned}
\int_{\Omega}\left(|v|^{\theta}+|u|^{\varrho}\right)|v|^{q+1} d x \leq & \int_{\Omega}|v|^{\theta+q+1} d x+\int_{\Omega}|u|^{\varrho}|v|^{q+1} d x \\
\leq & \int_{\Omega}|v|^{\theta+q+1} d x+\frac{\varrho}{\varrho+q+1} \delta_{2}^{\frac{\varrho+q+1}{\varrho}} \int_{\Omega}|u|^{\varrho+q+1} d x \\
& +\frac{q+1}{\varrho+q+1} \delta_{2}^{-\frac{\varrho+q+1}{q+1}} \int_{\Omega}|v|^{\varrho+q+1} d x \\
= & \|v\|_{\theta+q+1}^{\theta+q+1}+\frac{\varrho}{\varrho+q+1} \delta_{2}^{\frac{\varrho+q+1}{\varrho}}\|u\|_{\varrho+q+1}^{\varrho+q+1} \\
& +\frac{q+1}{\varrho+q+1} \delta_{2}^{-\frac{\varrho+q+1}{q+1}}\|v\|_{\varrho+q+1}^{\varrho+q+1} .
\end{aligned}
$$

Substituting (3.18) and (3.19) into (3.17), we have

$$
\begin{aligned}
\Psi^{\prime}(t) \geq & ((1-\sigma)-K \varepsilon) H^{-\sigma}(t) H^{\prime}(t)+\varepsilon(m+2)\left(\left\|u_{t}\right\|^{2}+\left\|v_{t}\right\|^{2}\right)+\varepsilon b_{1} m\left(\|\nabla u\|^{2}+\|\nabla v\|^{2}\right) \\
& +2 \varepsilon(m+1) H(t)+\varepsilon c^{\prime}\left(\|u\|_{2(r+2)}^{2(r+2)}+\|v\|_{2(r+2)}^{2(r+2)}\right) \\
& -\varepsilon \frac{k_{1}^{-p} H^{\sigma p}(t)}{p+1}\left(\|u\|_{k+p+1}^{k+p+1}+\frac{l}{l+p+1} \delta_{1}^{\frac{l+p+1}{l}}\|v\|_{l+p+1}^{l+p+1}+\frac{p+1}{l+p+1} \delta_{1}^{-\frac{l+p+1}{p+1}}\|u\|_{l+p+1}^{l+p+1}\right)
\end{aligned}
$$




$$
\begin{aligned}
& -\varepsilon \frac{k_{2}^{-q} H^{\sigma q}(t)}{q+1}\left(\|v\|_{\theta+q+1}^{\theta+q+1}+\frac{\varrho}{\varrho+q+1} \delta_{2}^{\frac{\varrho+q+1}{\varrho}}\|u\|_{\varrho+q+1}^{\varrho+q+1}\right. \\
& \left.+\frac{q+1}{\varrho+q+1} \delta_{2}^{-\frac{\varrho+q+1}{q+1}}\|v\|_{\varrho+q+1}^{\varrho+q+1}\right) .
\end{aligned}
$$

Since $2(r+2)>\max \{k+p+1, l+p+1, \theta+q+1, \varrho+q+1\}$, we obtain

$$
\begin{aligned}
& H^{\sigma p}(t)\|u\|_{k+p+1}^{k+p+1} \leq C\left(\|u\|_{2(r+2)}^{2 \sigma p(r+2)+k+p+1}+\|v\|_{2(r+2)}^{2 \sigma p(r+2)}\|u\|_{k+p+1}^{k+p+1}\right), \\
& H^{\sigma q}(t)\|v\|_{\theta+q+1}^{\theta+q+1} \leq C\left(\|v\|_{2(r+2)}^{2 \sigma q(r+2)+\theta+q+1}+\|u\|_{2(r+2)}^{2 \sigma q(r+2)}\|v\|_{\theta+q+1}^{\theta+q+1}\right), \\
& \frac{l}{l+p+1} \delta_{1}^{l+p+1}{ }^{l+1} H^{\sigma p}(t)\|v\|_{l+p+1}^{l+p+1} \\
& \quad \leq C \frac{l}{l+p+1} \delta_{1}^{\frac{l+p+1}{l}}\left(\|v\|_{2(r+2)}^{2 \sigma p(r+2)+l+p+1}+\|u\|_{2(r+2)}^{2 \sigma p(r+2)}\|v\|_{l+p+1}^{l+p+1}\right),
\end{aligned}
$$

and

$$
\begin{aligned}
& \frac{\varrho}{\varrho+q+1} \delta_{2}^{\frac{\varrho+q+1}{\varrho}} H^{\sigma q}(t)\|u\|_{\varrho+q+1}^{\varrho+q+1} \\
& \quad \leq C \frac{\varrho}{\varrho+q+1} \delta_{2}^{\frac{\varrho+q+1}{\varrho}}\left(\|u\|_{2(r+2)}^{2 \sigma q(r+2)+\varrho+q+1}+\|v\|_{2(r+2)}^{2 \sigma q(r+2)}\|u\|_{\varrho+q+1}^{\varrho+q+1}\right) .
\end{aligned}
$$

By using (3.11) and the algebraic inequality

$$
z^{v} \leq z+1 \leq\left(1+\frac{1}{a}\right)(z+a), \quad \forall z \geq 0,0<v \leq 1, a \geq 0,
$$

we have, for all $t \geq 0$,

$$
\begin{aligned}
\|u\|_{2(r+2)}^{2 \sigma p(r+2)+k+p+1} & \leq d\left(\|u\|_{2(r+2)}^{2(r+2)}+H(0)\right) \\
& \leq d\left(\|u\|_{2(r+2)}^{2(r+2)}+H(t)\right), \\
\|v\|_{2(r+2)}^{2 \sigma q(r+2)+\theta+q+1} & \leq d\left(\|v\|_{2(r+2)}^{2(r+2)}+H(t)\right),
\end{aligned}
$$

where $d=1+\frac{1}{H(0)}$. Similarly

$$
\begin{aligned}
& \|u\|_{2(r+2)}^{2 \sigma q(r+2)+\varrho+q+1} \leq d\left(\|u\|_{2(r+2)}^{2(r+2)}+H(t)\right), \\
& \|v\|_{2(r+2)}^{2 \sigma p(r+2)+l+p+1} \leq d\left(\|v\|_{2(r+2)}^{2(r+2)}+H(t)\right) .
\end{aligned}
$$

Also, since $(a+b)^{\lambda} \leq C\left(a^{\lambda}+b^{\lambda}\right), a, b>0$, by the Young inequality and using (3.11) and (3.25), we conclude that

$$
\begin{aligned}
\|v\|_{2(r+2)}^{2 \sigma p(r+2)}\|u\|_{k+p+1}^{k+p+1} & \leq C\left(\|v\|_{2(r+2)}^{2(r+2)}+\|u\|_{k+p+1}^{2(r+2)}\right) \\
& \leq C\left(\|v\|_{2(r+2)}^{2(r+2)}+\|u\|_{2(r+2)}^{2(r+2)}\right), \\
\|u\|_{2(r+2)}^{2 \sigma q(r+2)}\|v\|_{\theta+q+1}^{\theta+q+1} & \leq C\left(\|u\|_{2(r+2)}^{2(r+2)}+\|v\|_{2(r+2)}^{2(r+2)}\right), \\
\|u\|_{2(r+2)}^{2 \sigma p(r+2)}\|v\|_{l+p+1}^{l+p+1} & \leq C\left(\|u\|_{2(r+2)}^{2(r+2)}+\|v\|_{2(r+2)}^{2(r+2)}\right)
\end{aligned}
$$


and

$$
\|v\|_{2(r+2)}^{2 \sigma q(r+2)}\|u\|_{\varrho+q+1}^{\varrho+q+1} \leq C\left(\|v\|_{2(r+2)}^{2(r+2)}+\|u\|_{2(r+2)}^{2(r+2)}\right) .
$$

Combining (3.21)-(3.24) and (3.26)-(3.33) into (3.20), we have

$$
\begin{aligned}
\Psi^{\prime}(t) \geq & ((1-\sigma)-K \varepsilon) H^{-\sigma}(t) H^{\prime}(t)+\varepsilon(m+2)\left(\left\|u_{t}\right\|^{2}+\left\|v_{t}\right\|^{2}\right)+\varepsilon b_{1} m\left(\|\nabla u\|^{2}+\|\nabla v\|^{2}\right) \\
& +\varepsilon\left[2(m+1)-C k_{1}^{-p}\left(1+\frac{l}{l+p+1} \delta_{1}^{\frac{l+p+1}{l}}+\frac{p+1}{l+p+1} \delta_{1}^{-\frac{l+p+1}{p+1}}\right)\right. \\
& \left.-C k_{2}^{-q}\left(1+\frac{\varrho}{\varrho+q+1} \delta_{2}^{\frac{\varrho+q+1}{\varrho}}+\frac{q+1}{\varrho+q+1} \delta_{2}^{-\frac{\varrho+q+1}{q+1}}\right)\right] H(t) \\
& +\varepsilon\left[c^{\prime}-C k_{1}^{-p}\left(1+\frac{l}{l+p+1} \delta_{1}^{\frac{l+p+1}{l}}+\frac{p+1}{l+p+1} \delta_{1}^{-\frac{l+p+1}{p+1}}\right)\right. \\
& \left.-C k_{2}^{-q}\left(1+\frac{\varrho}{\varrho+q+1} \delta_{2}^{\frac{\varrho+q+1}{\varrho}}+\frac{q+1}{\varrho+q+1} \delta_{2}^{-\frac{\varrho+q+1}{q+1}}\right)\right] \\
& \times\left(\|u\|_{2(r+2)}^{2(r+2)}+\|v\|_{2(r+2)}^{2(r+2)}\right) .
\end{aligned}
$$

At this point, and for large values of $k_{1}$ and $k_{2}$, we can find positive constants $K_{1}$ and $K_{2}$ such that (3.34) becomes

$$
\begin{aligned}
\Psi^{\prime}(t) \geq & ((1-\sigma)-K \varepsilon) H^{-\sigma}(t) H^{\prime}(t)+\varepsilon(m+2)\left(\left\|u_{t}\right\|^{2}+\left\|v_{t}\right\|^{2}\right)+\varepsilon b_{1} m\left(\|\nabla u\|^{2}+\|\nabla v\|^{2}\right) \\
& +\varepsilon K_{1} H(t)+\varepsilon K_{2}\left(\|u\|_{2(r+2)}^{2(r+2)}+\|v\|_{2(r+2)}^{2(r+2)}\right) \\
\geq & \beta\left(\left\|u_{t}\right\|^{2}+\left\|v_{t}\right\|^{2}+H(t)+\|\nabla u\|^{2}+\|\nabla v\|^{2}+\|u\|_{2(r+2)}^{2(r+2)}+\|v\|_{2(r+2)}^{2(r+2)}\right),
\end{aligned}
$$

where $\beta=\min \left\{\varepsilon(m+2), \varepsilon b_{1} m, \varepsilon K_{1}, \varepsilon K_{2}\right\}$, and we pick $\varepsilon$ small enough so that $(1-\sigma)-$ $K \varepsilon \geq 0$. Consequently, we have

$$
\Psi(t) \geq \Psi(0)=H^{1-\sigma}(0)+\varepsilon\left(\int_{\Omega} u_{0} u_{1} d x+\int_{\Omega} v_{0} v_{1} d x\right)>0, \quad \forall t \geq 0 .
$$

On the other hand, applying the Hölder inequality, we obtain

$$
\begin{aligned}
\left|\int_{\Omega} u u_{t} d x+\int_{\Omega} v v_{t} d x\right|^{\frac{1}{1-\sigma}} & \leq\|u\|^{\frac{1}{1-\sigma}}\left\|u_{t}\right\|^{\frac{1}{1-\sigma}}+\|v\|^{\frac{1}{1-\sigma}}\left\|v_{t}\right\|^{\frac{1}{1-\sigma}} \\
& \leq C\left(\|u\|_{2(r+2)}^{\frac{1}{1-\sigma}}\left\|u_{t}\right\|^{\frac{1}{1-\sigma}}+\|v\|_{2(r+2)}^{\frac{1}{1-\sigma}}\left\|v_{t}\right\|^{\frac{1}{1-\sigma}}\right)
\end{aligned}
$$

The Young inequality gives

$$
\left|\int_{\Omega} u u_{t} d x+\int_{\Omega} v v_{t} d x\right|^{\frac{1}{1-\sigma}} \leq C\left(\|u\|_{2(r+2)}^{\frac{\mu_{1}}{1-\sigma}}+\left\|u_{t}\right\|^{\frac{\mu_{2}}{1-\sigma}}+\|v\|_{2(r+2)}^{\frac{\mu_{1}}{1-\sigma}}+\left\|v_{t}\right\|^{\frac{\mu_{2}}{1-\sigma}}\right)
$$

for $\frac{1}{\mu_{1}}+\frac{1}{\mu_{2}}=1$. We take $\mu_{2}=2(1-\sigma)$ to get $\mu_{1}=\frac{2(1-\sigma)}{1-2 \sigma}$ by (3.11). Therefore (3.38) becomes

$$
\left|\int_{\Omega} u u_{t} d x+\int_{\Omega} v v_{t} d x\right|^{\frac{1}{1-\sigma}} \leq C\left(\left\|u_{t}\right\|^{2}+\left\|v_{t}\right\|^{2}+\|u\|_{2(r+2)}^{\frac{2}{1-2 \sigma}}+\|v\|_{2(r+2)}^{\frac{2}{1-2 \sigma}}\right) .
$$


By using Lemma 2.3, we obtain

$$
\begin{aligned}
& \left|\int_{\Omega} u u_{t} d x+\int_{\Omega} v v_{t} d x\right|^{\frac{1}{1-\sigma}} \\
& \quad \leq C\left(\left\|u_{t}\right\|^{2}+\left\|v_{t}\right\|^{2}+\|u\|_{2(r+2)}^{2(r+2)}+\|v\|_{2(r+2)}^{2(r+2)}+\|\nabla u\|^{2}+\|\nabla v\|^{2}\right) .
\end{aligned}
$$

Thus

$$
\begin{aligned}
\Psi^{\frac{1}{1-\sigma}}(t) & =\left[H^{1-\sigma}(t)+\varepsilon\left(\int_{\Omega} u u_{t} d x+\int_{\Omega} v v_{t} d x\right)\right]^{\frac{1}{1-\sigma}} \\
& \leq 2^{\frac{\sigma}{1-\sigma}}\left(H(t)+\varepsilon^{\frac{1}{1-\sigma}}\left|\int_{\Omega} u u_{t} d x+\int_{\Omega} v v_{t} d x\right|^{\frac{1}{1-\sigma}}\right) \\
& \leq C\left(\left\|u_{t}\right\|^{2}+\left\|v_{t}\right\|^{2}+H(t)+\|u\|_{2(r+2)}^{2(r+2)}+\|v\|_{2(r+2)}^{2(r+2)}+\|\nabla u\|^{2}+\|\nabla v\|^{2}\right) .
\end{aligned}
$$

By combining (3.35) and (3.41), we arrive at

$$
\Psi^{\prime}(t) \geq \xi \Psi^{\frac{1}{1-\sigma}}(t)
$$

where $\xi$ is a positive constant.

A simple integration of $(3.42)$ over $(0, t)$ yields $\Psi^{\frac{\sigma}{1-\sigma}}(t) \geq \frac{1}{\Psi^{-\frac{\sigma}{1-\sigma}}(0)-\frac{\xi \sigma t}{1-\sigma}}$, which implies that the solution blows up in a finite time $T^{*}$ with

$$
T^{*} \leq \frac{1-\sigma}{\xi \sigma \Psi^{\frac{\sigma}{1-\sigma}}(0)} .
$$

\section{Competing interests}

The author declares that they have no competing interests.

Received: 12 November 2014 Accepted: 17 February 2015 Published online: 27 February 2015

\section{References}

1. Rammaha, MA, Sakuntasathien, S: Global existence and blow up of solutions to systems of nonlinear wave equations with degenerate damping and source terms. Nonlinear Anal. 72, 2658-2683 (2010)

2. Agre, K, Rammaha, MA: Systems of nonlinear wave equations with damping and source terms. Differ. Integral Equ. 19, 1235-1270 (2006)

3. Alves, CO, Cavalcanti, MM Domingos Cavalcanti, VN, Rammaha, MA, Toundykov, D: On the existence, uniform decay rates and blow up of solutions to systems of nonlinear wave equations with damping and source terms. Discrete Contin. Dyn. Syst., Ser. S 2, 583-608 (2009)

4. Houari, BS: Global nonexistence of positive initial-energy solutions of a system of nonlinear wave equations with damping and source terms. Differ. Integral Equ. 23, 79-92 (2010)

5. Houari, BS: Global existence and decay of solutions of a nonlinear system of wave equations. Appl. Anal. 91, 475-489 (2012)

6. Wu, J, Li, S, Chai, S: Existence and nonexistence of a global solution for coupled nonlinear wave equations with damping and source. Nonlinear Anal. 72(11), 3969-3975 (2010)

7. Fei, L, Hongjun, G: Global nonexistence of positive initial-energy solutions for coupled nonlinear wave equations with damping and source terms. Abstr. Appl. Anal. 2011, 760209 (2011)

8. Payne, LE, Sattinger, DH: Saddle points and instability of nonlinear hyperbolic equations. Isr. J. Math. 22(3-4), 273-303 (1975)

9. Vitillaro, E: Global nonexistence theorems for a class of evolution equations with dissipation. Arch. Ration. Mech. Anal. 149, 155-182 (1999)

10. Pişkin, E, Polat, N: Global existence, decay and blow up solutions for coupled nonlinear wave equations with damping and source terms. Turk. J. Math. 37, 633-651 (2013)

11. Messaoudi, SA, Houari, BS: Global nonexistence of positive initial-energy solutions of a system of nonlinear viscoelastic wave equations with damping and source terms. J. Math. Anal. Appl. 365, 277-287 (2010)

12. Adams, RA, Fournier, JJF: Sobolev Spaces. Academic Press, New York (2003)

13. Messaoudi, SA: Blow up in a nonlinearly damped wave equation. Math. Nachr. 231, 105-111 (2001) 